\title{
Functional and nutritional outcomes after gastric cancer surgery
}

\author{
Masahiro Tsujiura ${ }^{1,2}$, Souya Nunobe ${ }^{1}$ \\ ${ }^{1}$ Department of Surgery, Saiseikai Shiga Hospital, Ritto City, Japan; ${ }^{2}$ Department of Gastroenterological Surgery, Gastroenterological Center, \\ Cancer Institute Hospital, Japanese Foundation for Cancer Research, Koto-ward, Tokyo, Japan \\ Contributions: (I) Conception and design: M Tsujiura; (II) Administrative support: M Tsujiura; (III) Provision of study materials or patients: All \\ authors; (IV) Collection and assembly of data: M Tsujiura; (V) Data analysis and interpretation: All Authors; (VI) Manuscript writing: All authors; (VII) \\ Final approval of manuscript: All authors. \\ Correspondence to: Souya Nunobe, MD, PhD. Department of Gastroenterological Surgery, Gastroenterological Center, Cancer Institute Hospital, \\ Japanese Foundation for Cancer Research, 3-8-31 Ariake, Koto-ward, Tokyo 135-8550, Japan. Email: souya.nunobe@jfcr.or.jp.
}

\begin{abstract}
Recent improvements in diagnostic techniques and national screening programs have resulted in increasing number of patients diagnosed with early gastric cancer (EGC). The low incidence rate of lymph node metastasis and excellent survival rates after surgical treatment for EGC enabled the reduction in the extent of lymphadenectomy and the range of gastric resection for function-preserving gastrectomy. Thus, the quality of life (QOL) of patients with gastric cancer (GC) in the curative stage can be maintained. Moreover, these function-preserving procedures have been widely performed by less invasive procedures, such as laparoscopic and robotic approaches. Pylorus-preserving gastrectomy (PPG) and proximal gastrectomy (PG) represent the two main function-preserving surgical procedures for GC. PPG is an alternative to distal gastrectomy (DG) for cT1 N0 EGC located in the middle part of the stomach. Preservation of the pyloric function is expected to prevent post-gastrectomy syndromes such as dumping syndrome. PG is an alternative to total gastrectomy (TG) and can thus be performed for cT1 N0 EGC located in the upper part of the stomach. Preservation of the residual stomach is expected to work as a reservoir for ingested food. The optimal reconstruction method after PG among the three most commonly performed procedures (esophagogastrostomy, jejunal interposition, and double-tract reconstruction) remains controversial. In addition to these three reconstruction methods, the novel double-flap technique (DFT) of esophagogastrostomy has gained attention recently because of its potential usefulness to prevent postoperative esophageal reflux. In this review article, we summarize the current evidence of PPG and PG with esophagogastrostomy by the DFT, focusing on postoperative nutrition and QOL.
\end{abstract}

Keywords: Function-preserving gastrectomy; gastric cancer (GC); proximal gastrectomy (PG); esophagogastrostomy with double-flap technique (esophagogastrostomy with DFT); pylorus-preserving gastrectomy (PPG)

Received: 03 March 2019; Accepted: 07 November 2019; Published: 05 April 2020.

doi: $10.21037 / \operatorname{tgh} .2019 .11 .10$

View this article at: http://dx.doi.org/10.21037/tgh.2019.11.10

\section{Introduction}

Advancements in diagnostic techniques and population screening systems in high incidence countries, mainly in Japan and South Korea have resulted in detection of increasing numbers of early gastric cancer (EGC). The low rates of lymph node metastasis and excellent longterm survival after surgical treatment for EGC $(1,2)$ has enabled function-preserving gastrectomy, such as pyloruspreserving gastrectomy (PPG) and proximal gastrectomy (PG) which reduces the extent of lymphadenectomy and gastric resection (3-5). Moreover, these function-preserving procedures have been widely performed as laparoscopic and robotic approaches with the aim of maintaining patients' postoperative quality of life (QOL). Studies utilizing patient questionnaires have demonstrated the 


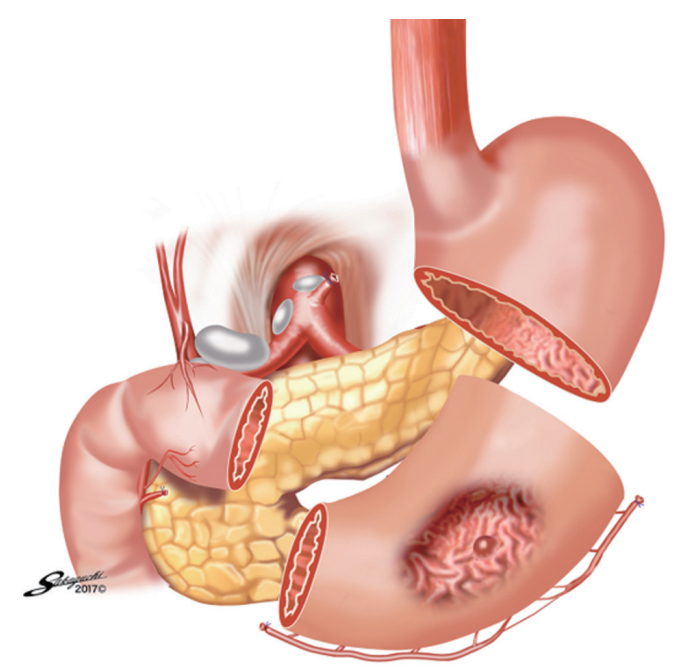

Figure 1 Surgical concept of PPG for GC in the middle stomach. The proximal remnant stomach is transected on the demarcation line between the right and left gastroepiploic arteries. The distal stomach is divided 4 to $5 \mathrm{~cm}$ proximal to the pyloric ring. The supra-/infra-pyloric vessels and the pyloric branch of the vagus nerve are preserved to maintain the blood supply and function of the pyloric cuff. PPG, pylorus-preserving gastrectomy; GC, gastric cancer. Reproduced from ref (4).

nutritional and symptomatic benefits of several techniques of function-preserving gastrectomy. In this review article, we present the current evidence of PPG and PG with esophagogastrostomy by the double-flap technique (DFT), focusing on postoperative nutrition and QOL.

\section{PPG}

PPG was initially applied to the treatment of benign gastric ulcers in 1967 (6). Since then, PPG has been widely used as a function-preserving procedure for the treatment of EGC (7). By preserving the pyloric ring, PPG is expected to possess several functional and nutritional merits with a lower incidence of post-gastrectomy syndromes, such as bile reflux and dumping syndrome, in comparison with conventional distal gastrectomy (DG) with Billroth I reconstruction (B1).

\section{Indications for PPG}

As described in the current version of the Japanese Gastric
Cancer Treatment Guidelines, PPG is a modified surgery for cT1N0 GC located in the middle portion of the stomach (8). Our basic indications for PPG are (I) a preoperative diagnosis of intramucosal or submucosal carcinoma without lymphatic metastasis; (II) a tumor located in the middle third of the stomach and $>5 \mathrm{~cm}$ away from the pyloric ring; (III) any histological type; (IV) patient age of $\leq 75$ years; and (V) no hiatal hernia or esophageal reflux (9). In our institution, patients with gastric cardia dysfunction or difficult dietary restrictions do not meet the criteria for PPG because their high risk of postoperative esophageal reflux and subsequent pulmonary aspiration.

\section{Laparoscopic procedures of PPG (Figure 1)}

The details of our laparoscopic procedures for PPG have been described previously $(10,11)$. For patients who met the indications for PPG at our institution, we performed D1+ lymphadenectomy, which includes lymph node stations $\# 1 / \# 3 \mathrm{a} / \# 3 \mathrm{~b} / \# 4 \mathrm{sb} / \# 4 \mathrm{~d} / \# 6 / \# 7 / \# 8 \mathrm{a} / \# 9$ as recommended in the Japanese Gastric Cancer Treatment Guidelines (8). During PPG, the infra-pyloric artery was routinely preserved; its vein was also preserved in surgeries performed after August 2012 to retain venous return in the pyloric cuff (12). The right gastroepiploic vessels were transected after bifurcation of the infra-pyloric vessels, so lymph node dissection of the infra-pyloric area (\#6i) was achieved with some limitation (13). The root of the right gastric artery and vein and the supra-pyloric lymph nodes (\#5) were routinely left intact, so these vessels were transected after the first branch. The hepatic and pyloric branches of the vagus nerve were routinely preserved, and its celiac branch was preserved in some cases (14).

Gastro-gastro anastomosis was performed either extracorporeally $(15)$ or intracorporeally $(16,17)$ for reconstruction during laparoscopic PPG (LPPG). More recently, we preferentially performed intracorporeal anastomosis using our newly established end-to-end gastrogastrostomy technique (17).

\section{Oncological safety of PPG}

As mentioned previously, the supra-/infra-pyloric vessels are preserved during PPG to sustain the blood supply and function of the pyloric cuff. Therefore, safety concerns about possible lymph node metastasis in these areas may 
arise. Previous reports have already shown relatively low incidence rates of supra-/infra-pyloric lymphatic metastasis, ranging from $0.00 \%$ to $0.90 \%$ (lymph node station \#5) and from $0.45 \%$ to $4.80 \%$ (lymph node station \#6), for early GC located in the middle part of the stomach $(13,18-20)$. Meticulous examination of the supra-/infra-pyloric areas is also important during surgery, and if necessary, it is recommended that sampled \#5 and \#6 lymph nodes will be sent for intraoperative pathological examination. This can further guide a surgeon's decision to convert to DG. In our experience, there were 2 of 475 conversions to DG $(0.42 \%)$ in patients for whom LPPG was initially planned between 2006 and 2012 (9).

Another oncological safety concern associated with PPG is the location of the resection lines of the stomach on both the proximal and distal sides. To secure negative margins, the extent of the primary lesion should be accurately diagnosed in the preoperative examinations, including biopsy results. Preoperative placement of marking clips along with intraoperative gastroscopy is considered to be very useful, especially in the laparoscopic approach. Additionally, frozen sections of the resection edges can be helpful in identifying the histological cancer-free margin during the surgery. Several reports have revealed satisfactory long-term survival rates of PPG (95.0-98.4\% overall 5-year survival rate) (9,21-24). The survival rate after PPG has also been proven comparable with that after DG $(20,21)$.

\section{Functional and nutritional outcomes after PPG (Table 1)}

The findings of previous reports of PPG and LPPG, focusing on nutritional/functional outcomes, are summarized in Table 1. The main advantage of PPG is the prevention of post-gastrectomy syndromes such as dumping syndrome and bile reflux. Other advantages include a wellmaintained postoperative body weight and nutritional status. Because PPG is an alternative to DG for EGC in the middle third of the stomach, several studies have compared the surgical results of PPG versus DG mainly by B1 (DG-B1). The sizes of the proximal stomach remnant and pyloric cuff were also discussed in some reports. The impact of some branches of the vagus nerve and the venous return of the pyloric cuff were also evaluated.

\section{Single-arm analysis $(9,15,25,26)$}

Favorable symptomatic and nutritional outcomes after PPG have been shown by some groups, including ours. The postoperative body weight was well-maintained after PPG, and the rate of body weight loss reportedly ranged from $6.0 \%$ to $6.8 \%$ among relatively large sample-size analyses.

\section{Superiority over DG-B1 (20,23,27-36)}

As mentioned above, several studies have compared surgical results between PPG and DG-B1. Because of pyloric ring preservation, $\mathrm{PPG}$ tends to be associated with lower rates of bile reflux and remnant gastritis and higher rates of food residue in endoscopic findings. In a survey of patient's postoperative symptoms, PPG showed a lower rate of dumping syndrome, as expected; however, it tended to have higher rates of abdominal fullness/distention. Although the results differed among individual reports, PPG showed superiority over DG-B1 in postoperative gallstone formation, body weight changes, and nutritional parameters $(20,29,30,33)$.

\section{QOL analysis according to the postgastrectomy} syndrome assessment scale-45 (PGSAS-45) $(37,38)$

The PGSAS- 45 is a questionnaire examination used to assess post-gastrectomy-specific clinical symptoms and QOL, developed by the Japan Postgastrectomy Syndrome Working Party (43). Two multi-center analyses have revealed significantly better outcomes regarding dumping syndrome and diarrhea after PPG, compared with DG.

\section{The size of remnant stomach (39-41)}

Namikawa et al. compared the QOL scores according to the size of the proximal gastric remnant using the PGASA-45, resulting in no significant differences in symptoms of reflux, dumping and diarrhea (39). The size of the pyloric cuff generally showed no definitive impact on the remnant stomach or patient symptoms per both endoscopic findings and symptom survey, respectively.

\section{Preservation of branches of the vagus nerve $(14,42)$}

Preservation of the hepatic and pyloric branches significantly reduced the incidence rate of postoperative gallstone formation (42). Because the pyloric and hepatic branches are routinely preserved in our institution, we analyzed the influence of preserving the celiac branch of the vagus nerve (14). We found no definite impact of preservation of the celiac branch of the vagus nerve.

\section{Preservation of the infra-pyloric vein (12)}

Preservation of the infra-pyloric vein can help to prevent 


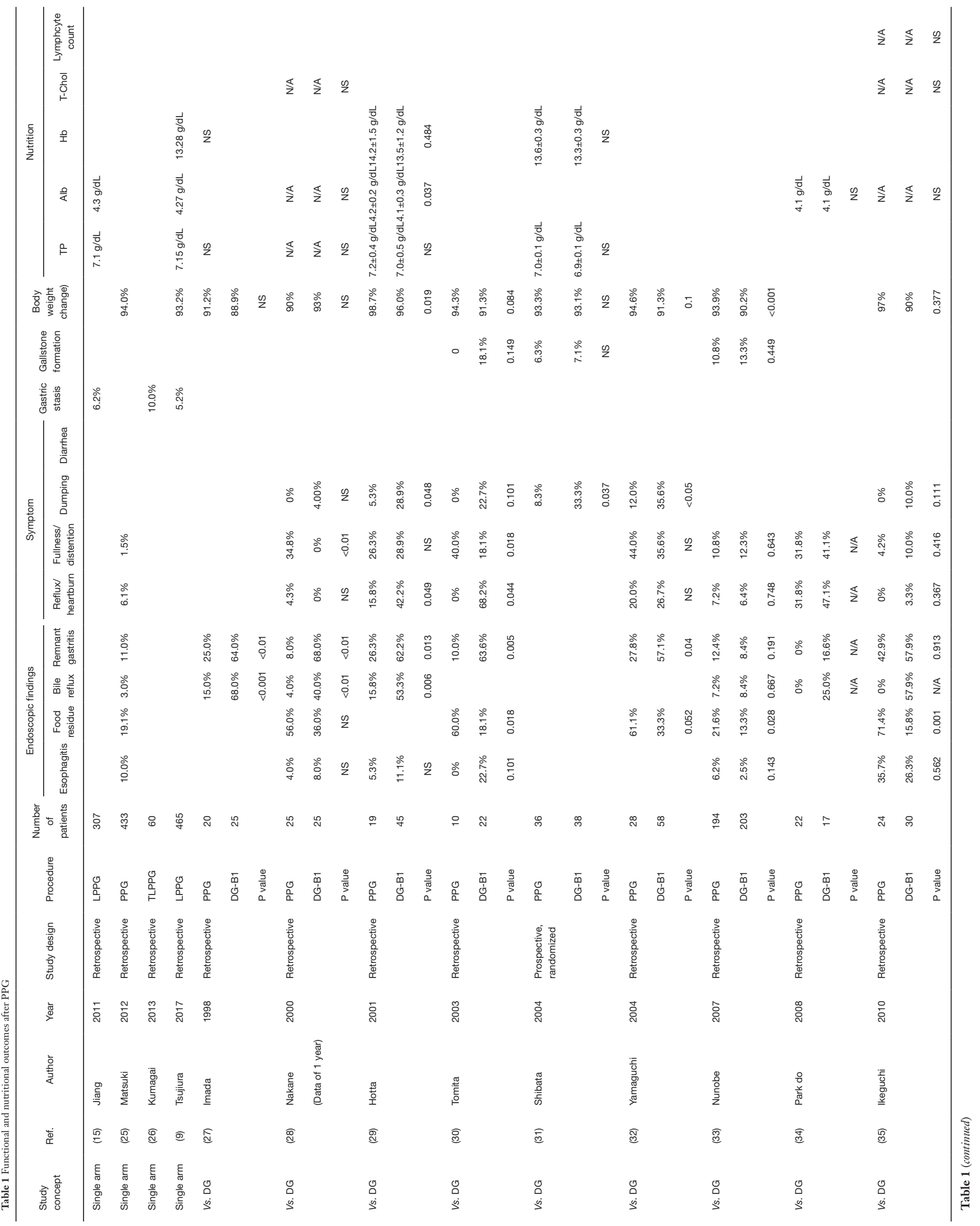




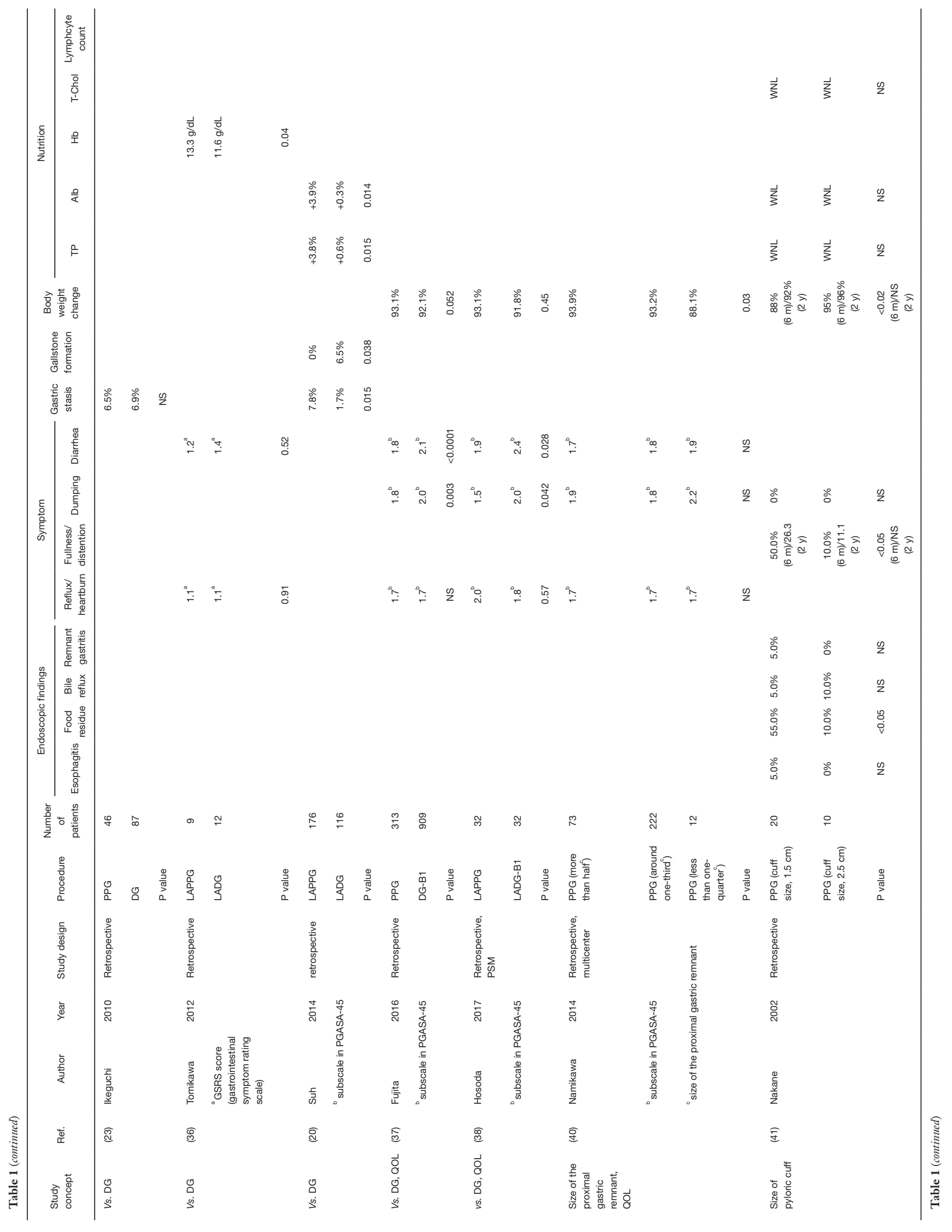




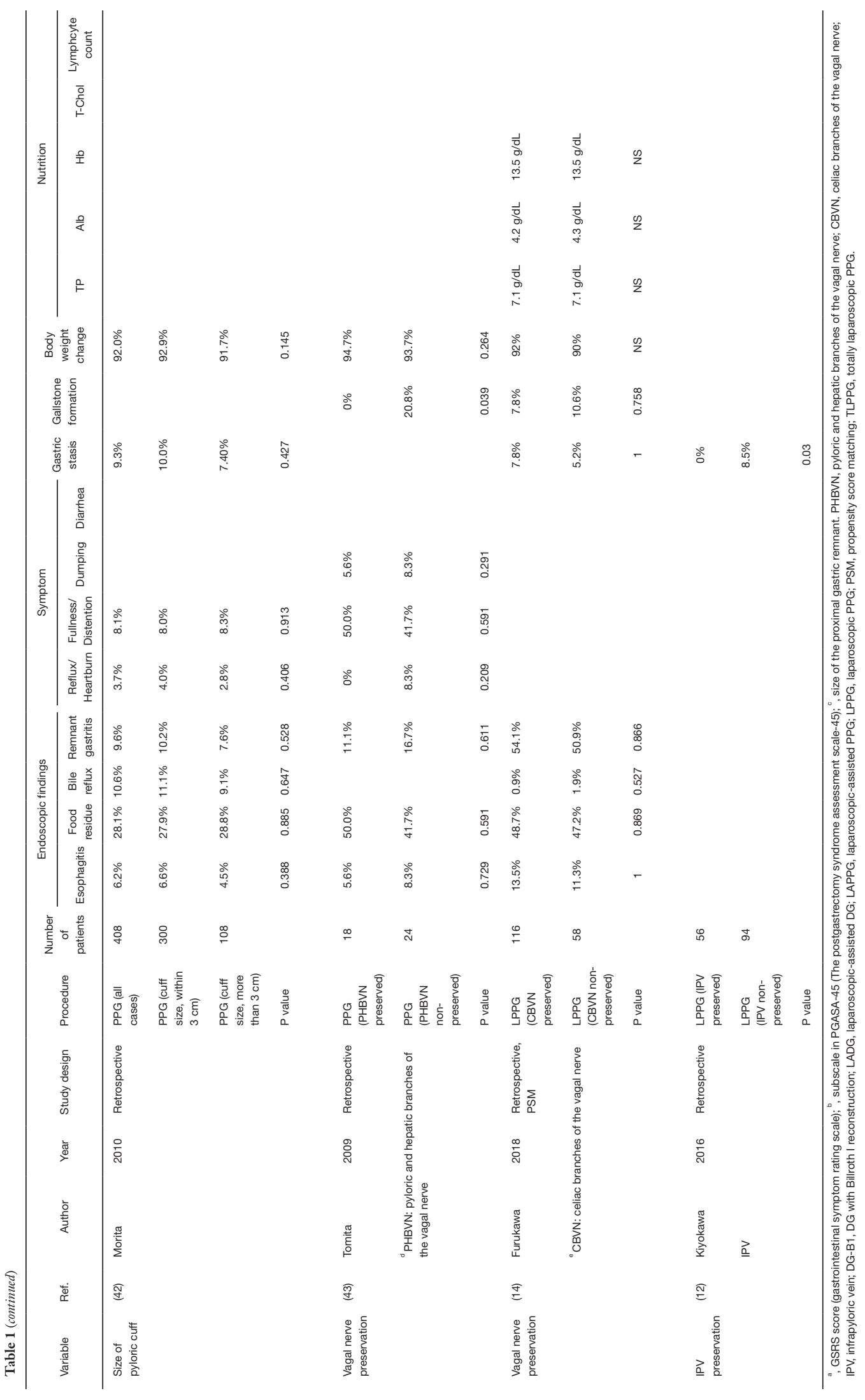


postoperative gastric stasis after LPPG by reducing venous stasis and edema of the pyloric cuff. Therefore, the infrapyloric vein has been preserved in all patients treated since August 2012 in our institution. In Korea, the ongoing randomized controlled trial "KLASS 04 " is comparing postoperative QOL and surgical outcomes between LPPG and laparoscopic DG (44). The results are expected to expound on the potential advantages of PPG in the near future.

\section{PG with esophagogastrostomy by DFT}

PG is an alternative procedure to total gastrectomy (TG) for cT1 cN0 GC in the upper part of the stomach as described in the Japanese Gastric Cancer Treatment Guidelines (8). Because of the growing trend of the incidence of proximal GC (45), the demand for PG is increasing. In terms of reconstruction after PG, three major procedures have been described: esophagogastrostomy, jejunal interposition, and double-tract reconstruction. These three procedures have their respective pros and cons, and the optimal reconstruction method continues to be controversial $(5,46,47)$.

Esophagogastrostomy is the simplest reconstruction procedure after PG; however, it is associated with a risk of reflux esophagitis and anastomotic stenosis. A novel esophagogastrostomy method with the DFT, first reported by Kamikawa et al. (48) in 2001, is a hand-sewn procedure that very effectively prevents postoperative reflux. In recent years, several reports have shown the potential advantages of esophagogastrostomy with the DFT. In the present study, we focused on this promising reconstruction method and summarized its surgical outcomes and functional/nutritional advantages.

\section{Laparoscopic procedures of PG with esophagogastrostomy by DFT}

The details of our laparoscopic procedures for PG have been described previously $(4,49)$. D1+ lymphadenectomy which includes lymph node stations \#1/\#2/\#3a/\#4sa/\#4sb/ $\# 7 / \# 8 \mathrm{a} / \# 9 / \# 11 \mathrm{p}$ was performed as recommended in the Japanese Gastric Cancer Treatment Guidelines (8). The right gastric and right gastroepiploic vessels were routinely preserved during PG. The hepatic and pyloric branches of the vagus nerve were routinely preserved, and its celiac branch was preserved in some cases.
Intraoperative gastroscopy was performed in all cases of laparoscopic PG (LPG). During the endoscopy, the locations of the esophagogastric junction, primary lesion, and preoperatively placed marking clips were confirmed to secure proper resection margins during surgery. In some cases, an intraoperative frozen section of the resection edge was examined to identify the histological cancer-free margin.

The remnant stomach was extracted from the umbilical port site and the seromuscular double-flaps $(2.5 \mathrm{~cm}$ wide $\times 3.5 \mathrm{~cm}$ high) were created on the anterior wall using electric cautery (Figure 2A). After creation of the double flaps, the gastric mucosa was opened at the inferior edge for anastomosis. The posterior wall of the esophagus was fixed to the superior edge of the mucosal window (Figure 2B, red arrows). Continuous suturing was applied between all layers of the posterior esophageal wall and the superior opening of the mucosa on the gastric remnant (Figure 2B). Layerby-layer anastomosis was performed between the anterior wall of the esophagus and the inferior opening of the gastric wall by interrupted or continuous sutures (Figure 2C). Finally, the esophagogastric anastomosis was fully covered by seromuscular flaps, and the completed anastomosis was Y-shaped (Figure 2D).

\section{Functional and nutritional outcomes after PG with esophagogastrostomy by DFT (Table 2)}

Previous reports of esophagogastrostomy by the DFT are summarized in Table 2 (49-56). This new DFT technique was first described by Kamikawa et al. (48) in 2001, and its clinical and surgical outcomes have been published in the English-language literature since 2015. Most of these reports describe the surgical results for GC located in the upper stomach; a few reports describe the results for esophagogastric junctional cancer $(50,56)$. Mine et al. (50) performed this reconstruction method for esophagogastric junctional cancers and indicated its potential usefulness for intrathoracic anastomosis.

Most previous reports are retrospective, small-scale, and single-arm analyses; however, they have shown extremely lower incidence rates of anastomotic complications and gastroesophageal reflux compared with other types of esophagogastrostomy $(5,46,47)$. In a large-sample analysis, Kuroda et al. (56) accumulated surgical outcomes of more than 500 DFT cases from multiple institutes between 1996 and 2005. The authors reported low incidence 

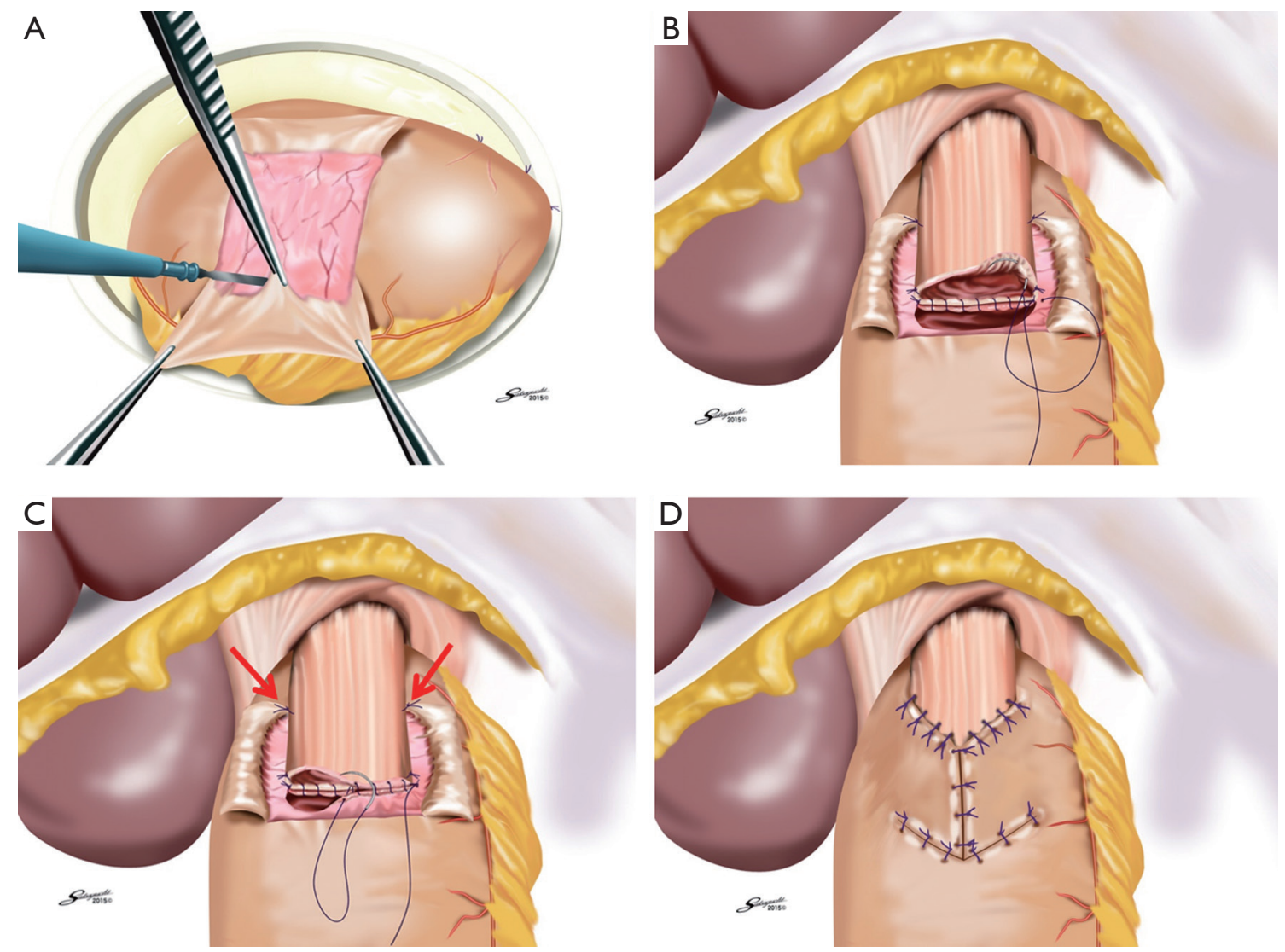

Figure 2 Esophagogastrostomy with double-flap technique (DFT). (A) Creation of the seromuscular double flaps $(2.5 \mathrm{~cm}$ wide $\times 3.5 \mathrm{~cm}$ high) on the anterior wall of the remnant stomach. (B) Fixation between the posterior wall of the esophagus and the superior edge of the mucosal window (red arrows). Suturing between all layers of the posterior esophageal wall and superior opening of the mucosa on the gastric remnant. (C) Suturing between the anterior wall of the esophagus and the inferior opening of the gastric wall. (D) Coverage of the esophagogastric anastomosis by seromuscular flaps. The completed anastomosis is Y-shaped. Reproduced from ref (4).

rates of not only anastomotic complications $(7.2 \%$ of all anastomosis-related complications), but also severe reflux esophagitis (only $6.0 \%$ of grade B or higher by the Los Angeles classification), suggesting a safe and steady antireflux mechanism of DFT. With respect to anastomotic complications, the incidence rates of anastomotic leakage are relatively low, ranging from $0.0 \%$ to $7.7 \%$. However, the rates of anastomotic stricture are reportedly as high as $29.1 \%$. Shibasaki et al. (54) showed the negative relationship between anastomotic stenosis and the total number of stitches. In performing this DFT technique, an excessive number of stiches should be avoided because of the possibility of anastomotic stenosis.

The complexity of the DFT is another one of its negative aspects. Shibasaki et al. (54) performed this procedure using a robotic approach and reported a shorter and more acceptable anastomotic time than that achieved by a laparoscopic approach. The usage of knotless barbed absorbable suture may also effectively shorten the anastomotic time (55).

Limited comparison between PG and its alternative technique TG exists (49). Our analysis confirmed that there are several advantages of laparoscopic PG-DFT over laparoscopic TG with Roux-en-Y reconstruction. These superior outcomes of PG over TG include the lower incidence rates of postoperative complications, shorter postoperative hospital stay, and better nutritional status. Level I evidence in support of DFT procedure is expected as prospective studies or randomized clinical trials with a large sample size are performed. 


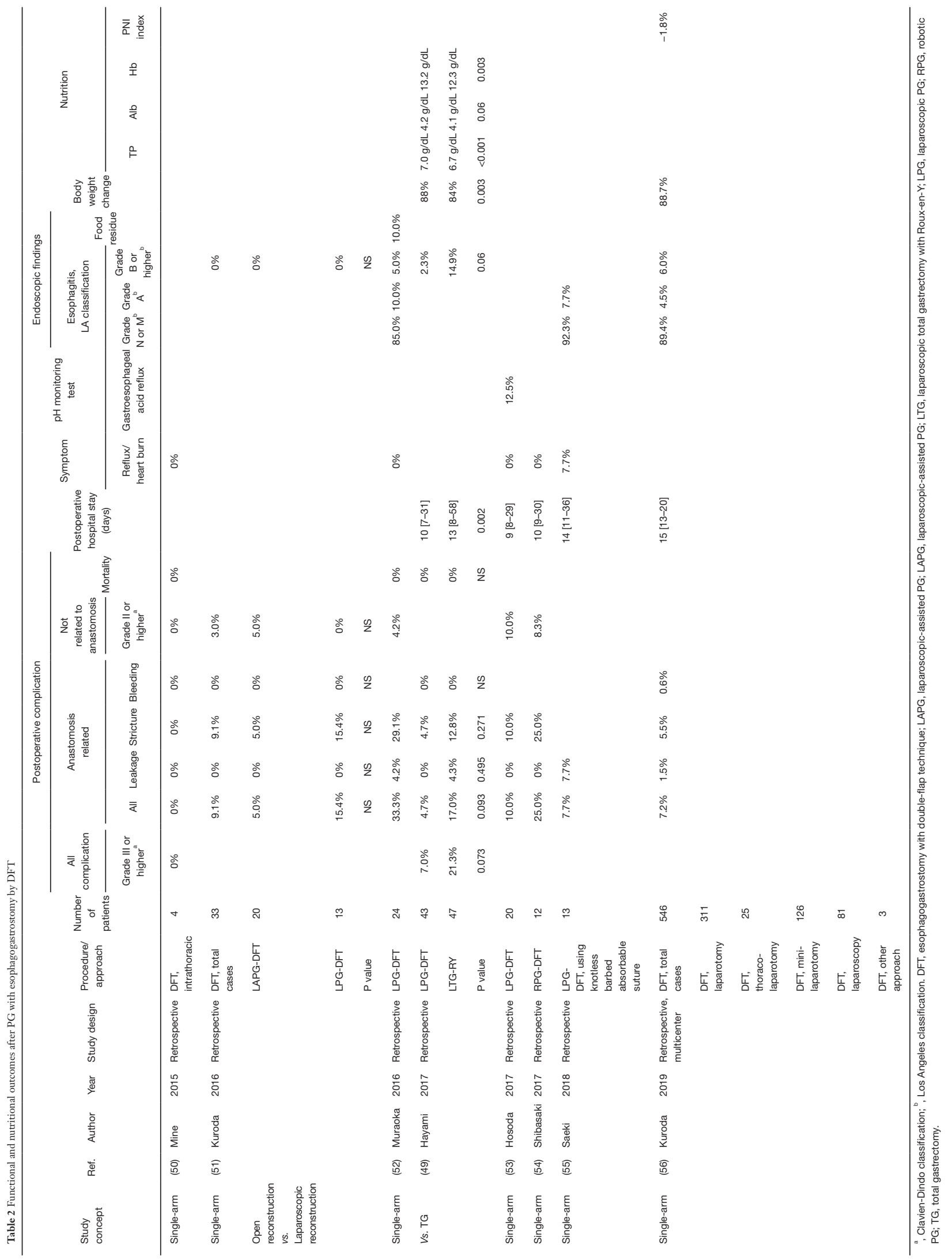




\section{Acknowledgments}

We thank Angela Morben, DVM, ELS, from Edanz Group (www.edanzediting.com/ac), for editing a draft of this manuscript.

\section{Footnote}

Conflicts of Interest: The authors have no conflicts of interest to declare.

Ethical Statement: The authors are accountable for all aspects of the work in ensuring that questions related to the accuracy or integrity of any part of the work are appropriately investigated and resolved.

\section{References}

1. Nashimoto A, Akazawa K, Isobe $\mathrm{Y}$, et al. Gastric cancer treated in 2002 in Japan: 2009 annual report of the JGCA nationwide registry. Gastric Cancer 2013;16:1-27.

2. Sano T, Hollowood A. Early gastric cancer: diagnosis and less invasive treatments. Scand J Surg 2006;95:249-55.

3. Hiki N, Nunobe S, Kubota T, et al. Function-preserving gastrectomy for early gastric cancer. Ann Surg Oncol 2013;20:2683-92.

4. Nunobe S, Hiki N. Function-preserving surgery for gastric cancer: current status and future perspectives. Transl Gastroenterol Hepatol 2017;2:77.

5. Ueda Y, Shiroshita H, Etoh T, et al. Laparoscopic proximal gastrectomy for early gastric cancer. Surg Today 2017;47:538-47.

6. Maki T, Shiratori T, Hatafuku T, et al. Pylorus-preserving gastrectomy as an improved operation for gastric ulcer. Surgery 1967;61:838-45.

7. Yokota T, Ishiyama S, Saito T, et al. Treatment strategy of limited surgery in the treatment guidelines for gastric cancer in Japan. Lancet Oncol 2003;4:423-8.

8. Japanese Gastric Cancer Association. Japanese gastric cancer treatment guidelines 2014 (ver. 4). Gastric Cancer 2017;20:1-19.

9. Tsujiura M, Hiki N, Ohashi M, et al. Excellent Long-Term Prognosis and Favorable Postoperative Nutritional Status After Laparoscopic Pylorus-Preserving Gastrectomy. Ann Surg Oncol 2017;24:2233-40.

10. Fukunaga T, Hiki N, Kubota T, et al. Oncologic outcomes of laparoscopy-assisted distal gastrectomy for gastric cancer. Ann Surg Oncol 2013;20:2676-82.
11. Hiki N, Kaminishi M. Pylorus-preserving gastrectomy in gastric cancer surgery--open and laparoscopic approaches. Langenbecks Arch Surg 2005;390:442-7.

12. Kiyokawa T, Hiki N, Nunobe S, et al. Preserving infrapyloric vein reduces postoperative gastric stasis after laparoscopic pylorus-preserving gastrectomy. Langenbecks Arch Surg 2017;402:49-56.

13. Mizuno A, Shinohara H, Haruta S, et al. Lymphadenectomy along the infrapyloric artery may be dispensable when performing pylorus-preserving gastrectomy for early middle-third gastric cancer. Gastric Cancer 2017;20:543-7.

14. Furukawa H, Ohashi $M$, Honda $M$, et al. Preservation of the celiac branch of the vagal nerve for pyloruspreserving gastrectomy: is it meaningful? Gastric Cancer 2018;21:516-23.

15. Jiang X, Hiki N, Nunobe S, et al. Postoperative outcomes and complications after laparoscopy-assisted pyloruspreserving gastrectomy for early gastric cancer. Ann Surg 2011;253:928-33.

16. Kumagai K, Hiki N, Nunobe S, et al. Totally laparoscopic pylorus-preserving gastrectomy for early gastric cancer in the middle stomach: technical report and surgical outcomes. Gastric Cancer 2015;18:183-7.

17. Ohashi M, Hiki N, Ida S, et al. A novel method of intracorporeal end-to-end gastrogastrostomy in laparoscopic pylorus-preserving gastrectomy for early gastric cancer, including a unique anastomotic technique: piercing the stomach with a linear stapler. Surg Endosc 2018;32:4337-43.

18. Kim BH, Hong SW, Kim JW, et al. Oncologic safety of pylorus-preserving gastrectomy in the aspect of micrometastasis in lymph nodes at stations 5 and 6 . Ann Surg Oncol 2014;21:533-8.

19. Kong SH, Kim JW, Lee HJ, et al. The safety of the dissection of lymph node stations 5 and 6 in pyloruspreserving gastrectomy. Ann Surg Oncol 2009;16:3252-8.

20. Suh YS, Han DS, Kong SH, et al. Laparoscopy-assisted pylorus-preserving gastrectomy is better than laparoscopyassisted distal gastrectomy for middle-third early gastric cancer. Ann Surg 2014;259:485-93.

21. Aizawa M, Honda M, Hiki N, et al. Oncological outcomes of function-preserving gastrectomy for early gastric cancer: a multicenter propensity score matched cohort analysis comparing pylorus-preserving gastrectomy versus conventional distal gastrectomy. Gastric Cancer 2017;20:709-17. 
22. Hiki N, Sano T, Fukunaga T, et al. Survival benefit of pylorus-preserving gastrectomy in early gastric cancer. J Am Coll Surg 2009;209:297-301.

23. Ikeguchi M, Hatada T, Yamamoto M, et al. Evaluation of a pylorus-preserving gastrectomy for patients preoperatively diagnosed with early gastric cancer located in the middle third of the stomach. Surg Today 2010;40:228-33.

24. Morita S, Katai H, Saka M, et al. Outcome of pyloruspreserving gastrectomy for early gastric cancer. Br J Surg 2008;95:1131-5.

25. Matsuki A, Nashimoto A, Yabusaki H, et al. Long-term clinical outcome and survival after pylorus-preserving gastrectomy. Hepatogastroenterology 2012;59:2012-5.

26. Kumagai K, Hiki N, Nunobe S, et al. Totally laparoscopic pylorus-preserving gastrectomy for early gastric cancer in the middle stomach: technical report and surgical outcomes. Gastric Cancer 2015;18:183-7.

27. Imada T, Rino Y, Takahashi M, et al. Postoperative functional evaluation of pylorus-preserving gastrectomy for early gastric cancer compared with conventional distal gastrectomy. Surgery 1998;123:165-70.

28. Nakane Y, Akehira K, Inoue K, et al. Postoperative evaluation of pylorus-preserving gastrectomy for early gastric cancer. Hepatogastroenterology 2000;47:590-5.

29. Hotta T, Taniguchi K, Kobayashi Y, et al. Postoperative evaluation of pylorus-preserving procedures compared with conventional distal gastrectomy for early gastric cancer. Surg Today 2001;31:774-9.

30. Tomita R, Fujisaki S, Tanjoh K. Pathophysiological studies on the relationship between postgastrectomy syndrome and gastric emptying function at 5 years after pyloruspreserving distal gastrectomy for early gastric cancer. World J Surg 2003;27:725-33.

31. Shibata C, Shiiba KI, Funayama Y, et al. Outcomes after pylorus-preserving gastrectomy for early gastric cancer: a prospective multicenter trial. World J Surg 2004;28:857-61.

32. Yamaguchi T, Ichikawa D, Kurioka H, et al. Postoperative clinical evaluation following pylorus-preserving gastrectomy. Hepatogastroenterology 2004;51:883-6.

33. Nunobe S, Sasako M, Saka M, et al. Symptom evaluation of long-term postoperative outcomes after pyloruspreserving gastrectomy for early gastric cancer. Gastric Cancer 2007;10:167-72.

34. Park DJ, Lee HJ, Jung HC, et al. Clinical outcome of pylorus-preserving gastrectomy in gastric cancer in comparison with conventional distal gastrectomy with Billroth I anastomosis. World J Surg 2008;32:1029-36. 35. Ikeguchi M, Kuroda H, Kihara K, et al. Nutritional assessment of patients after pylorus-preserving gastrectomy for early gastric cancer. Indian J Surg 2010;72:453-7.

36. Tomikawa M, Korenaga D, Akahoshi T, et al. Quality of life after laparoscopy-assisted pylorus-preserving gastrectomy: an evaluation using a questionnaire mailed to the patients. Surg Today 2012;42:625-32.

37. Fujita J, Takahashi M, Urushihara T, et al. Assessment of postoperative quality of life following pylorus-preserving gastrectomy and Billroth-I distal gastrectomy in gastric cancer patients: results of the nationwide postgastrectomy syndrome assessment study. Gastric Cancer 2016;19:302-11.

38. Hosoda K, Yamashita K, Sakuramoto S, et al. Postoperative quality of life after laparoscopy-assisted pylorus-preserving gastrectomy compared With laparoscopy-assisted distal gastrectomy: A cross-sectional postal questionnaire survey. Am J Surg 2017;213:763-70.

39. Namikawa T, Hiki N, Kinami S, et al. Factors that minimize postgastrectomy symptoms following pylorus-preserving gastrectomy: assessment using a newly developed scale (PGSAS-45). Gastric Cancer 2015;18:397-406.

40. Nakane Y, Michiura T, Inoue K, et al. Length of the antral segment in pylorus-preserving gastrectomy. Br J Surg 2002;89:220-4.

41. Morita S, Sasako M, Saka M, et al. Correlation between the length of the pyloric cuff and postoperative evaluation after pylorus-preserving gastrectomy. Gastric Cancer 2010;13:109-16.

42. Tomita R. Gastric emptying function in patients 5 years after pylorus-preserving distal gastrectomy with or without preserving pyloric and hepatic branches of the vagal nerve for early gastric cancer. World J Surg 2009;33:2119-26.

43. Nakada K, Ikeda M, Takahashi M, et al. Characteristics and clinical relevance of postgastrectomy syndrome assessment scale (PGSAS)-45: newly developed integrated questionnaires for assessment of living status and quality of life in postgastrectomy patients. Gastric Cancer 2015;18:147-58.

44. Lee JH. Ongoing surgical clinical trials on minimally invasive surgery for gastric cancer: Korea. Transl Gastroenterol Hepatol 2016;1:40.

45. Ahn HS, Lee HJ, Yoo MW, et al. Changes in clinicopathological features and survival after gastrectomy for gastric cancer over a 20-year period. Br J Surg 2011;98:255-60.

46. Nakamura M, Yamaue H. Reconstruction after proximal gastrectomy for gastric cancer in the upper third of the 
stomach: a review of the literature published from 2000 to 2014. Surg Today 2016;46:517-27.

47. Wang S, Lin S, Wang H, et al. Reconstruction methods after radical proximal gastrectomy: A systematic review. Medicine 2018;97:0000000000010121.

48. Kamikawa Y, Kobayashi T, Kamiyama S, et al. A new procedure of esophagogastrostomy to prevent reflux following proximal gastrectomy (in Japanese). Shoukakigeka 2001;24:1053-60.

49. Hayami M, Hiki N, Nunobe S, et al. Clinical Outcomes and Evaluation of Laparoscopic Proximal Gastrectomy with Double-Flap Technique for Early Gastric Cancer in the Upper Third of the Stomach. Ann Surg Oncol 2017;24:1635-42.

50. Mine S, Nunobe S, Watanabe M. A Novel Technique of Anti-reflux Esophagogastrostomy Following Left Thoracoabdominal Esophagectomy for Carcinoma of the Esophagogastric Junction. World J Surg 2015;39:2359-61.

51. Kuroda S, Nishizaki M, Kikuchi S, et al. DoubleFlap Technique as an Antireflux Procedure in Esophagogastrostomy after Proximal Gastrectomy. J Am Coll Surg 2016;223:e7-13.

doi: $10.21037 / \operatorname{tgh} .2019 .11 .10$

Cite this article as: Tsujiura $M$, Nunobe S. Functional and nutritional outcomes after gastric cancer surgery. Transl Gastroenterol Hepatol 2020;5:29.
52. Muraoka A, Kobayashi M, Kokudo Y. LaparoscopyAssisted Proximal Gastrectomy with the Hinged Double Flap Method. World J Surg 2016;40:2419-24.

53. Hosoda K, Yamashita K, Moriya H, et al. Laparoscopically Assisted Proximal Gastrectomy with Esophagogastrostomy Using a Novel "Open-Door" Technique : LAPG with Novel Reconstruction. J Gastrointest Surg 2017;21:1174-80.

54. Shibasaki S, Suda K, Nakauchi M, et al. Robotic valvuloplastic esophagogastrostomy using double flap technique following proximal gastrectomy: technical aspects and short-term outcomes. Surg Endosc 2017;31:4283-97.

55. Saeki Y, Tanabe K, Yamamoto Y, et al. Laparoscopic proximal gastrectomy with hinged double flap method using knotless barbed absorbable sutures: A case series. Int J Surg Case Rep 2018;51:165-9.

56. Kuroda S, Choda Y, Otsuka S, et al. Multicenter retrospective study to evaluate the efficacy and safety of the double-flap technique as antireflux esophagogastrostomy after proximal gastrectomy (rD-FLAP Study). Ann Gastroenterol Surg 2018;3:96-103. 